\title{
Fabrication of open-cellular (porous) titanium alloy implants: osseointegration, vascularization and preliminary human trials
}

\author{
Shujun $\mathrm{Li}^{1 \dagger}$, Xiaokang $\mathrm{Li}^{2 \dagger}$, Wentao Hou ${ }^{1}$, Krishna C. Nune ${ }^{3}$, R. Devesh K. Misra ${ }^{3}$, \\ Victor L. Correa-Rodriguez ${ }^{3}$, Zheng Guo ${ }^{2 *}$, Yulin $\mathrm{Hao}^{1}$, Rui Yang ${ }^{1}$ and Lawrence E. Murr ${ }^{3}$
}

\begin{abstract}
In this study we describe the fabrication of a variety of open-cellular titanium alloy (Ti-6Al-4V) implants, both reticular mesh and foam structures, using electron beam melting (EBM). These structures allow for the elimination of stress shielding by adjusting the porosity (or density) to produce an elastic modulus (or stiffness) to match that of both soft (trabecular) and hard (cortical) bone, as well as allowing for bone cell ingrowth, increased cell density, and all-matrix interactions; the latter involving the interplay between bone morphogenetic protein (BMP-2) and osteoblast functions. The early formation and characterization of elementary vascular structures in an aqueous hydrogel matrix are illustrated. Preliminary results for both animal (sheep) and human trials for a number of EBM-fabricated, and often patient-specific Tialloy implants are also presented and summarized. The results, while preliminary, support the concept and development of successful, porous, engineered "living" implants.
\end{abstract}

Keywords: porous implant fabrication, osseointegration, vascularization, animal and clinical human trials

\section{INTRODUCTION}

Although there have been several millions of successful human metal and alloy orthopedic implant installations of different kinds in the past decade [1], adverse issues continue to exist, including stress shielding (where the modulus or stiffness of the solid, fully dense implant metal is much greater than that of bone: $110 \mathrm{GPa}$ for Ti-6Al-4V alloy, in contrast to $20 \mathrm{GPa}$ for hard, cortical bone), aseptic loosening, infection, little or no bone cell ingrowth (os- seointegration) and no vascularization. However, advances in three-dimensional (3D) printing/additive manufacturing have demonstrated the ability to fabricate open-cellular (porous) implants that match bone stiffness and promote osseointegration, thereby eliminating the need for implant cement [2]. Porous implants fabricated by additive manufacturing techniques such as electron beam melting (EBM) or selective laser melting (SLM) have allowed for patient-specific metal or alloy implant manufacturing using computer-aided design (CAD) software incorporating patient micro-computed tomography (CT) $[3,4]$. These appliances, either variously configured reticulated mesh structures or foam structures, also allow for the incorporation of antibiotic agents [2,5] along with growth factors, signaling molecules, and specific cells promoting oxygen and nutrient exchange which facilitates efficient cell seeding and extracellular matrix (ECM) production; including tissue regeneration and vascularization [6-9]. Growth factors are proteins which serve as signaling agents for specific cell function such as bone morphogenetic proteins (BMPs) and vascular (endothelial) cell growth factor (VEGF) [10-11]. Kang et al. [12] have also recently noted that, "a challenge for tissue engineering is producing $3 \mathrm{D}$ vascularized cellular constructs of clinically relevant size, shape and structural integrity".

For nearly a decade, tens of thousands of EBM or SLM fabricated acetabular cups for hip implants, with designed porosity, have been surgically inserted into humans [13]. In addition, human trials of other manufactured ortho-

\footnotetext{
${ }^{1}$ Shenyang National Laboratory for Materials Science, Institute of Metal Research, Chinese Academy of Sciences, Shenyang 110016, China

${ }^{2}$ Department of Orthopedics, Xijing Hospital, Fourth Military Medical University, Xi'an 710032, China

${ }^{3}$ Department of Metallurgical, Materials and Biomedical Engineering, The University of Texas at El Paso, El Paso, TX 79968, USA

${ }^{\dagger}$ These authors contributed equally to this work.

* Corresponding author (email: guozheng@fmmu.edu.cn)
} 
pedic appliances have been performed by the US Army Walter Reed Medical Center in the US as well as Peking Third Hospital Orthopedic Department in China over the past five years.

In this study, we describe the fabrication of open cellular (mesh and foam) prototypes of Ti-6Al-4V alloy by EBM for fundamental studies of osseointegration and vascularization, along with more medically and patient-specific implant configurations. We discuss several animal and human trials for these implants. In particular, we investigate osteoblast cell proliferation and distribution throughout the EBM-fabricated structures as well as the initial stages of vascular (endothelial cell) structure in these alloy open cellular, with the intention of illustrating the creation of an engineered "living" implant.

\section{EXPERIMENTAL SECTION}

\section{Test sample and implant design and fabrication}

It has already been demonstrated that a variety of opencellular structures of Ti-6Al-4V alloy can be systematically fabricated using EBM, and mesh or foam unit cells embedded in appropriate CAD software $[2,3,14]$. In addition, metal and alloy mesh and foam structures fabricated by EBM have been described by the Gibson-Ashby equation [15]:

$$
E / E_{\mathrm{s}}=\left(\rho / \rho_{\mathrm{s}}\right)^{2},
$$

where $E$ is the modulus of elasticity (or the stiffness) for the fabricated, open-cellular structure having a density $\rho$, and $E_{\mathrm{s}}$ and $\rho_{\mathrm{s}}$ are the corresponding fully dense (solid) modulus and density, respectively. The percent porosity is also related to the relative density $\left(\rho / \rho_{\mathrm{s}}\right)$ by

$$
\operatorname{Porosity}(\%)=\left[1-\left(\frac{\rho}{\rho_{\mathrm{s}}}\right)\right] \times 100 .
$$

It can be observed from Equation (1) that the stiffness for an open-cellular structure implant can be tailored to that of bone, considering that $E_{\mathrm{s}}$ and $\rho_{\mathrm{s}}$ for Ti-6Al- $4 \mathrm{~V}$ are $110 \mathrm{GPa}$ and $4.43 \mathrm{~g} \mathrm{~cm}^{-3}$, respectively.

Fig. 1 illustrates the EBM system schematic characteristics of the $\mathrm{Arcam} \mathrm{Al}$ and $\mathrm{A} 2$ commercial systems used in this study for fabricating both cube-oriented (rhombic dodecahedron unit cell) mesh samples and various foam samples, and implant configurations. These systems use an electron beam accelerated to a potential of $60 \mathrm{kV}$, with beam currents adjusted according to the scan strategies which include a preheat scan sequence for each raked powder layer followed by a selective melt scan [14]. Fig. 2 shows the range of mesh and foam test samples having an

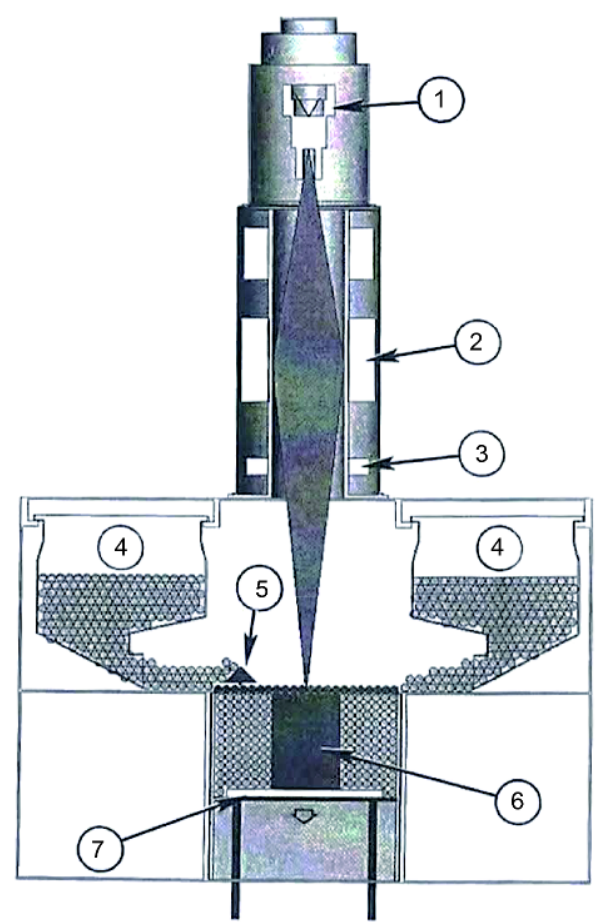

Figure 1 EBM system schematically showing the powder feed cassettes, layer raking, and electron beam scanning management (in vacuum). Electron gun is shown at (1), while (2) and (3) indicate the focus and scan lens and coils, respectively. The powder cassettes are indicated at (4), while (5) indicates the powder layer rake system. (6) and (7) illustrate the EBM product and build table, respectively.

elastic modulus ranging from 2 to $20 \mathrm{GPa}$, while Fig. 3 shows an example of porous Ti-6Al-4V alloy spine interbody fusion cages incorporating these open-cellular structures having densities, porosities, and elastic moduli indicated. These porous $\mathrm{Ti}$ alloy cages possess elastic modulus matching human bone, good bone-material integration and improved mechanical stability [16].

Cell culture, seeding efficiency, proliferation studies, and characterization

Cell culture studies were performed using mouse pre-osteoblast MC3T3-E1 subclone 4 cell line obtained from the American Type Cell Culture Collection (ATCC, Manassas, VA, USA). Alpha minimum essential medium ( $\alpha$-MEM, Invitrogen Corporation, USA) supplemented with $10 \%$ fetal bovine serum (FBS), penicillin $\left(100 \mathrm{U} \mathrm{mL}^{-1}\right)$ and streptomycin $\left(100 \mu \mathrm{g} \mathrm{mL}^{-1}\right)$ was used to culture the preosteoblasts. Open cellular 3D Ti-6Al-4V alloy mesh and foam structures were first subjected to a cleaning and disinfection procedure involving $1 \mathrm{~h}$ ultra-sonication cycles in detergent, followed by acetone, isopropanol, ethanol, and deionized water. The scaffolds were then sterilized 

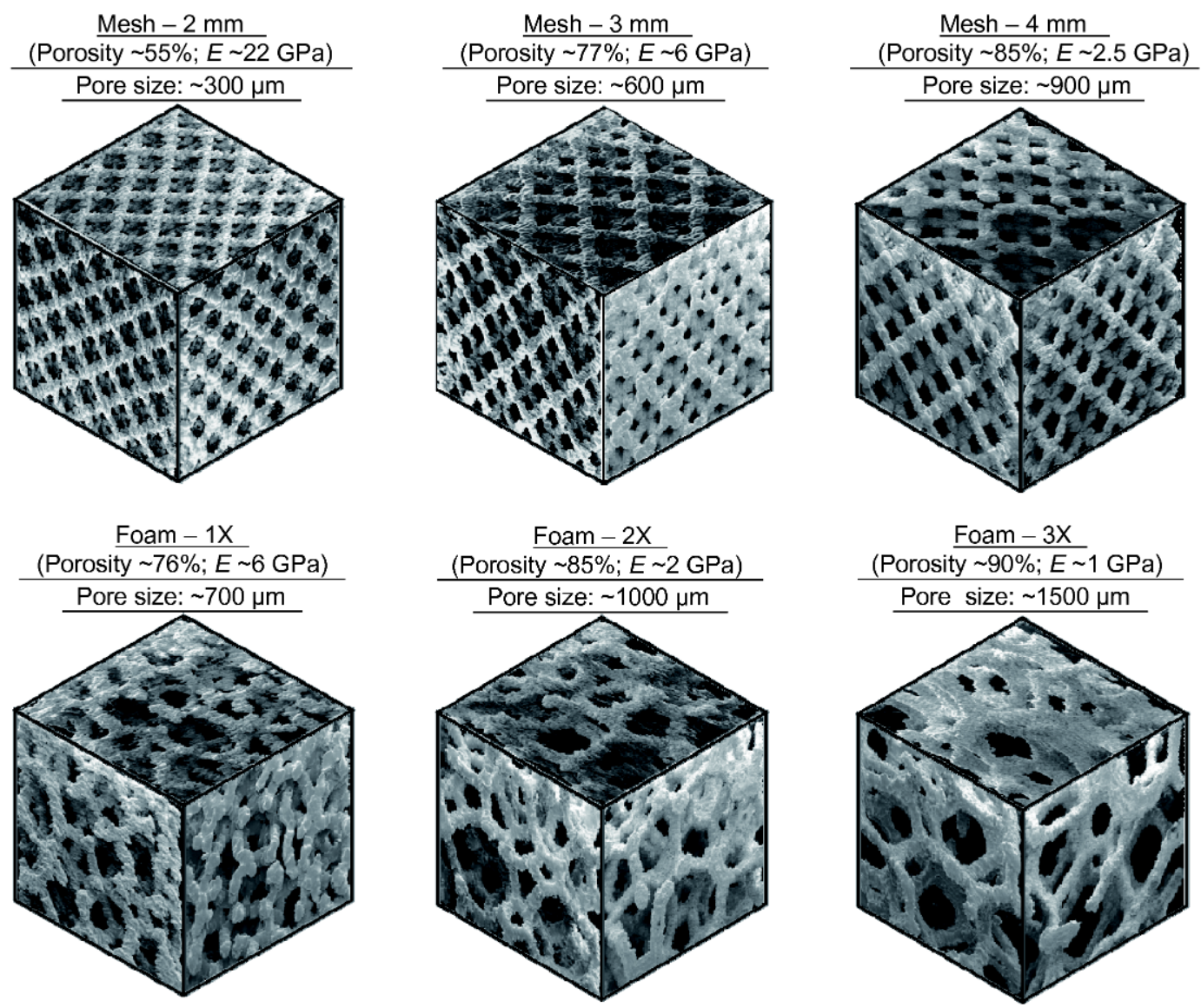

Figure 2 Examples of Ti-6Al-4V reticulated mesh and foam scaffolds used for the osseointegration and angiogenesis testing. Reprinted with permission from Ref. [22,23]. Copyright 2014, American Scientific Publishers, and Wiley, respectively.

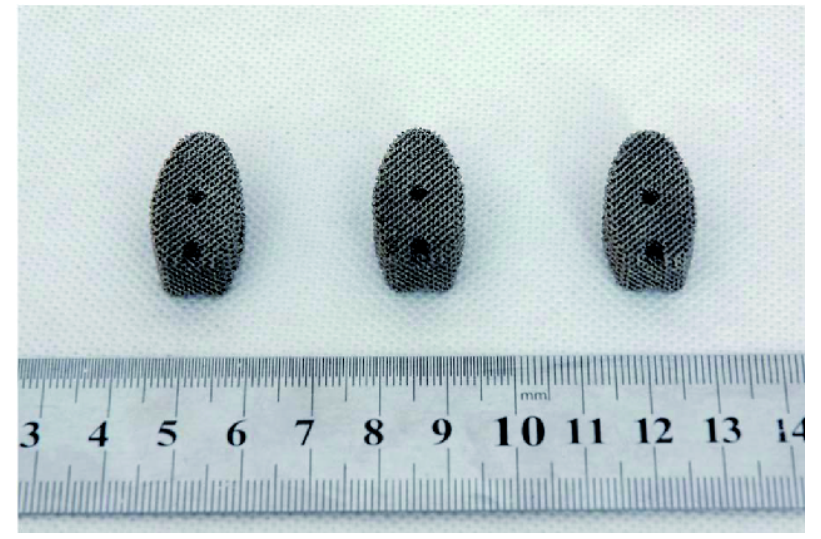

Figure 3 Examples of porous Ti-6Al-4V alloy spine interbody fusion cages fabricated by EBM. in an autoclave and dried overnight under UV light. Subsequently, the sterilized scaffolds were placed in 24well plate and incubated with cell suspension $\left(2 \times 10^{4}\right.$ cells/ scaffold) at $37^{\circ} \mathrm{C}$ in a humidified incubator with $5 \% \mathrm{CO}_{2}$ and $95 \%$ air.

For determining the cell seeding efficiency, mesh and foam titanium alloy structures were seeded with the preosteoblasts and incubated for $4 \mathrm{~h}$ at $37^{\circ} \mathrm{C}$ in a $\mathrm{CO}_{2}$ incubator. After the culture period, the specimens were removed from their respective wells and the cells remaining in each well were trypsinized, pelletized, resuspended, and stained with Trypan Blue dye for counting using a hemacytometer. The number of cells estimated from the hemacytometer was subtracted from the total number of initially seeded cells, to obtain the number of cells that were seeded on each specimen. The seeding efficiency was calculated using Equation (3):

Seeding efficiency $(\%)=\left(\frac{(\text { total number of cells seeded })-(\text { cells attached to the well })}{\text { total number of cells seeded }}\right) \times 100$. 
Cell proliferation rate per day was determined from the proliferation data measured through MTT (3-(4,5-dimethylthiazol-2-yl)-2,5-diphenyltetrazolium bromide) assay up to 14 days. Culture medium was changed every 2-3 days for the duration of the experiment. The assay is based on the reductase activity in mitochondria of living cells. These enzymes cleave the tetrazolium ring, which turns the pale yellow MTT into dark blue formazan crystals, the concentration of which is directly proportional to the number of metabolically active cells. After pre-determined incubation times, the samples were washed twice with phosphate buffer solution (PBS) and incubated with fresh culture medium containing MTT $\left(0.5 \mathrm{mg} \mathrm{mL}^{-1}\right.$ medium) at $37^{\circ} \mathrm{C}$ for $4 \mathrm{~h}$ in dark. Then, the unreacted dye was removed and dimethyl sulfoxide (DMSO) was added to dissolve the intracellular purple formazan product into a colored solution. The absorbance of this solution was quantified by spectrophotometer at $570 \mathrm{~nm}$ with a plate reader (Bio TEK Instrument, EL307C).

Scanning electron microscopy (SEM) was used to characterize the morphology, pore-interconnectivity, and in-growth of pre-osteoblasts grown on mesh and foam scaffolds for 21 days. The cell seeded scaffolds were fixed with $2.5 \%$ glutaraldehyde in $0.1 \mathrm{~mol} \mathrm{~L}^{-1}$ cacodylate buffer (pH 7.4) for $20 \mathrm{~min}$, rinsed with PBS, dehydrated with a graded series of ethanol (10\%-100\%) and dried. In order to evaluate the ingrowth of cells within the titanium mesh and foam structures in vitro, the specimens were sectioned along the $z$-axis after 21 days of cell culture period. The scaffolds were sputter-coated with $10 \mathrm{~nm}$ of gold and examined in a Hitachi S-4800N scanning electron microscope.

\section{Angiogenesis induction in matrix-infused metal foam}

A strategy for promoting osseointegration and vascularization in open-cellular, porous implants involves the infusion of the structure with a suitable bio-matrix such as a hydrogel containing collagen, proteins, pre-osteoblast cells, endothelial cells, appropriate signaling molecules, growth factors, and other function promoters such as hypoxia promoters. Hypoxia has been reported to increase cell viability and progression of survival signaling pathways that mediate aspects of endothelial, stromal, and vascular support cell biology; including vessel patterning [17]. Deferoxamine mesylate (DFM) (or deferoxamine (DFO)) promotes angiogenesis by inducing hypoxia $[18,19]$, and it has been reported that endothelial cells are able to form capillaries in gel when exposed to DFM. A hypoxia mimetic environment has been reported to enhance bone repair and endothelial integrity restoration
[20].

Human umbilical vein endothelial cell (HUVEC) membrane prepared by cell culture described in detail by Correa-Rodriguez et al. [21] was stained with $\mathrm{PKH} 26$ (Sigma-Aldrich red fluorescent dye). Similarly, mouse preosteoblast (MC3T3-E1) membrane was stained with PKH67 (Sigma-Aldrich green fluorescent dye). After confirming successful staining, the Ti-6Al-4V foam slices from fabricated samples similar to those shown in Fig. 2 $\left(0.5 \mathrm{~cm}\right.$ thick, having a density of $1.8 \mathrm{~g} \mathrm{~cm}^{-3} ; 60 \%$ porosity corresponding to an elastic modulus $E \sim 18 \mathrm{GPa}$ ) were seeded with $2 \times 10^{5}$ fluorescently labeled MC3T3-E1 cells and incubated for $12 \mathrm{~h}$ to allow cellular adherence as described above. This cell addition was done in order to test the hypoxic response of the pre-osteoblast cells to DFM. Matrigel (Corning Life Sciences Trademarked product), a hydrogel containing protein, collagen IV, heparin sulfate proteoglycans, entactin/nidogen, and growth factors, was added to the foam slices until the foam was completely covered. A suspension of the stained HUVECs in media was then treated with $2 \mathrm{mmol} \mathrm{L}^{-1} \mathrm{DFM}$ and added to cover the Matrigel-infused foam slices; and incubated at tissue culture incubator conditions for $18 \mathrm{~h}$ [21]. A similar experiment was also performed for the stained HUVECs in media not treated with DFM. These experimental (DFM-treated and untreated) foam monoliths were then observed using a Zeiss Axiovert 200 fluorescent microscope.

\section{Osseointegration of porous $\mathrm{Ti}-6 \mathrm{Al}-4 \mathrm{~V}$ cage}

The porous Ti-alloy cage, with dimensions of $\phi 12^{\prime} 5 \mathrm{~mm}$, possesses fully interconnected porous structure (porosity: $68 \pm 5.3 \%$; pore size: $710 \pm 42 \mu \mathrm{m}$ ) and a similar Young's modulus as natural bone $(2.5 \pm 0.2 \mathrm{GPa})$. Discectomy and cage implantation were applied at cervical disc levels (C3/ C4 or C4/C5), in 18 mature female Small Tail Han sheep (1-2 years old, $35-50 \mathrm{~kg}$ ). Anterior titanium plate was applied (CERVI-LOCK ${ }^{\mathrm{TM}}$, WEIGAO ORTHOPAEDIC DEVICE, China) to get an early stability. At three months, frontal and lateral X-rays were taken; six sheep were sacrificed for micro-CT and histological analyses by intravenous overdose of xylazine hydrochloride. The remaining sheep were sacrificed at six months for micro $\mathrm{CT}$ and histological analyses after frontal and lateral Xrays. The bone volume fraction (BVF) in the implant was calculated by dividing the volume of bone tissue in the implant by the total void volume of the implant. Mineralized Bone Fraction (MBF) was utilized to evaluate the bone fraction in the implant pores. 


\section{Clinical application of individualized porous Ti-alloy prosthesis}

A 35-year-old man had a gradually enlarging pelvic mass for nine months. The biopsy confirmed the diagnosis of pelvic giant cell tumor (GCT). The plain radiograph (Fig. 4a), CT scan (Fig. 4b, c) and MRI image (Fig. 4d) showed a lytic lesion on the right ischium and pubic bone. All image data were stored in Digital Imaging and Communication in Medical format (DICOM) and imported into CAD system for 3D tumor model reconstruction and prosthesis design. The process of image segmentation was conducted using the Mimics software (Leuven, Belgium). The bone was isolated from other tissues and structures such as muscle, fat, skin, and metal table of CT scanner. Pseudo color image was applied on DICOM image pixel data, and segmented images were used to reconstruct a 3D model. Under virtual condition, the tumor was simulated to be excised and the prosthesis was fixed at the defect site. The authors could rotate the 3D model to observe and refine the prosthesis design until the most appropriate one was selected. Finally, the safe margin, cutting plane, implant position, and screw sites were preoperatively planned and marked on the 3D model basing on surgical approach and exposure. The navigation-guided surgical resection was performed according to the pre-operation planning. The Ti-alloy prosthesis was prepared by EBM. The clinical application of the prothesis got the approvement of Independent Ethics Committee (IEC), First Affiliated Hospital of Fourth Military Medical University (Approval No: XJLL(2014)0023).

\section{RESULTS AND DISCUSSION}

\section{Seeding efficiency, cell proliferation, and cell adhesion}

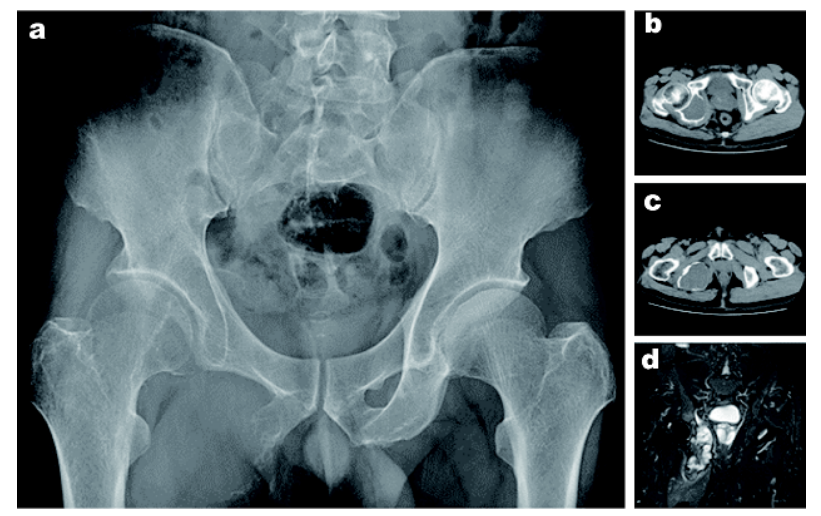

Figure 4 A 35-year-old male patient with periacetabular GCT. (a) X-ray film shows a mixed lytic lesion. (b, c) Axial CT images of the lesion. (d) Coronal images of T2-weighted MRI showing destructed ischium and surrounding soft tissue mass.
On both mesh and foam structures, as illustrated in Fig. 5a, the cell seeding efficiency was reduced with increasing porosity: $55 \%$ to $30 \%$ decrease in seeding efficiency with increasing porosity from $76 \%$ to $90 \%$ in the case of foam structures; $80 \%$ to $55 \%$ decrease in seeding efficiency with increasing porosity from $55 \%$ to $85 \%$ for mesh structures $[22,23]$. Penetration of cells through the interconnected pores, allows cells to attach to not only the struts inside the mesh, but also the underlying tissue culture wells. Thus, the seeding efficiency decreased with increase in porosity.

Cell proliferation increased with time on both mesh and foam structures. The proliferation rate was higher for 2 $\mathrm{mm}$ mesh (porosity $\sim 55 \%$; $E \sim 22 \mathrm{GPa}$ ) as compared to other structures studied (Fig. 5b). Proliferation of cells beyond two weeks led to a fibrous structure of ECM with
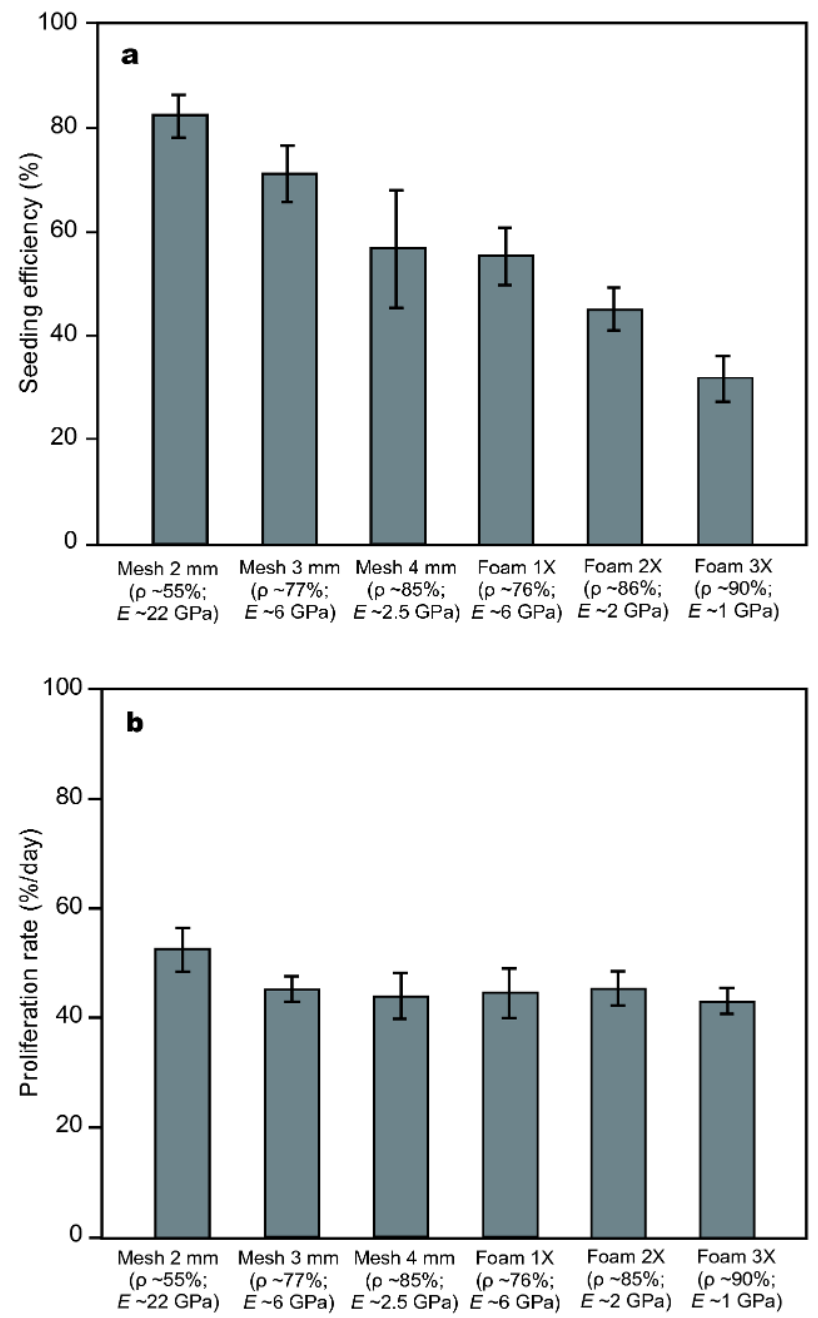

Figure 5 Histograms representing (a) seeding efficiency and (b) cell proliferation rate of pre-osteoblasts on 3D-porous Ti-6Al-4V alloy mesh and foam structures with different porosity (ranging from 55\% to $90 \%$ ) and elastic stiffness (ranging from $\sim 1$ to $22 \mathrm{GPa}$ ). 


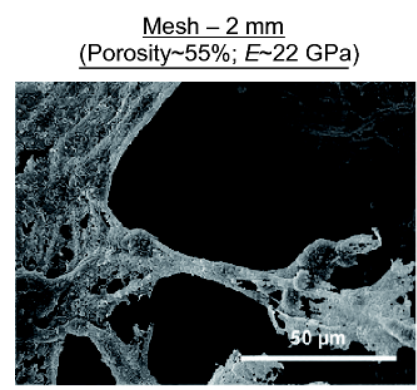

Foam - 1X (Porosity 76\%; E 6 GPa)

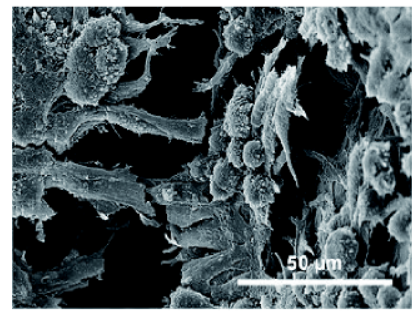

Mesh $-3 \mathrm{~mm}$ (Porosity 77\%; E 6 GPa)

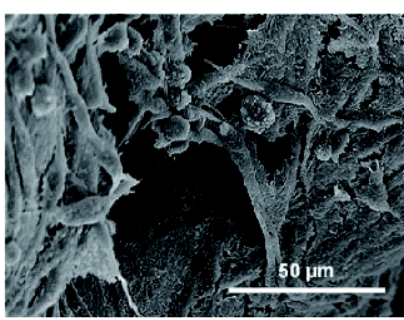

Foam - 2X (Porosity 85\%; E 2 GPa)

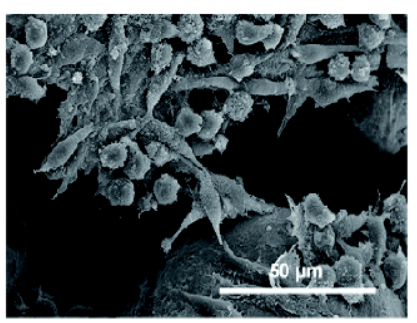

Mesh $-4 \mathrm{~mm}$ (Porosity 85\%; E 2.5GPa)

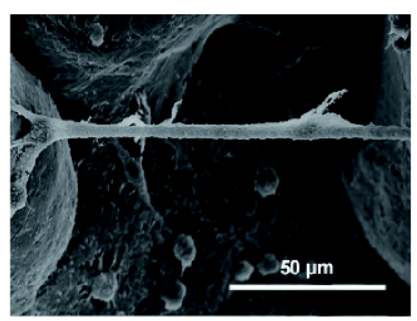

Foam - 3X (Porosity 90\%; E 1 GPa)

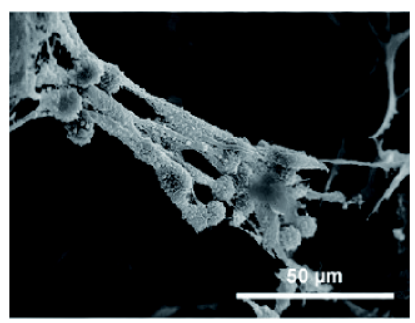

Figure 6 SEM images representing cellular in-growth and inter-connectivity of pre-osteoblasts (bridging the pores) on 3D-porous Ti-6Al-4V alloy mesh (top) and foam (bottom) structures with different porosity (ranging from 55\% to 90\%) and elastic stiffness (ranging from $~ 1$ to 22 GPa) after 21 days of incubation period.

cells wrapping and bridging the pores with their elongated morphology and 3D filo podia extensions along the struts and ligaments of the mesh and foam scaffolds (Fig. 6) [24]. The layer-by-layer organization of cells in the ECM along the struts and ligaments of the porous structure indicates the formation of osteoid like-matrix. Inter-cellular interaction in porous implant structures provides a pathway for cell communication and differentiated phenotype malfunction, leading to colonization of the entire structure. Phenotype maturity was also expressed by calcium mineralization (mineralized ECal) secreted by cells along the struts and ligaments of the porous structures revealed in previous studies using a differentiation assay [11].

Scaffolds with 3D interconnecting pore structure facilitated mechanical interlocking with the surrounding bone and promoted cell and tissue ingrowth (osteogenesis) by enabling proliferation of cells into the pores [24,25]. In our study, 3D porous titanium mesh and foam structures fabricated by EBM closely mimic the microarchitecture (spatial organization of cells and ECM molecules) of the in vivo bone. Furthermore, the unique microstructure of mesh and foam provided conditions that favored bone tissue formation by influencing cell attachment, proliferation and spreading. Moreover, the interconnecting pore architecture of the scaffolds facilitated circulation of physiological fluid, oxygen, and nutrients, and distribution of cells to the core of the biomedical implant.

\section{Angiogenesis induction}

Fig. 7 illustrates that angiogenesis is induced in endothelial cells through exposure to DFM which promotes capillarity in a 3D collagen based hydrogel (Matrigel) in a Ti-6Al-4V foam slice similar to previous observations of endothelial capillarity [20]. Fluorescent microscope images near the metal foam ligaments in the prepared slices, similar to Fig. 6, also illustrate the MC3T3-E1 cells exposed to DFM are proliferating and differentiating into phenotypes promoting structural bone component formation. This was observed earlier by measuring mineralized calcium which increased in the presence of DFM [21].

Similarly, le Noble et al. [26] have also suggested that DFM treatment helps restore bone formation defects by expansion of osteoprogenesis and osteoblasts. Correspondingly, Ramaswamy et al. [27] demonstrated that bone vascularization contains specialized endothelial cells with signaling properties supporting maturation and regeneration of bone. Fig. 8 illustrates the conceptual features for bone cell ingrowth and vascularization in a porous intramedullary metal rod in the femur. These observations support the prospects that porous metal implants infused with a suitable hydrogel containing requisite pre-osteoblast and endothelial cell concentrations along with DFM may support and accelerate vascular (primary capillary) structure development, bone ingrowth, and hydroxyapatite formation. In addition, as implicit in 

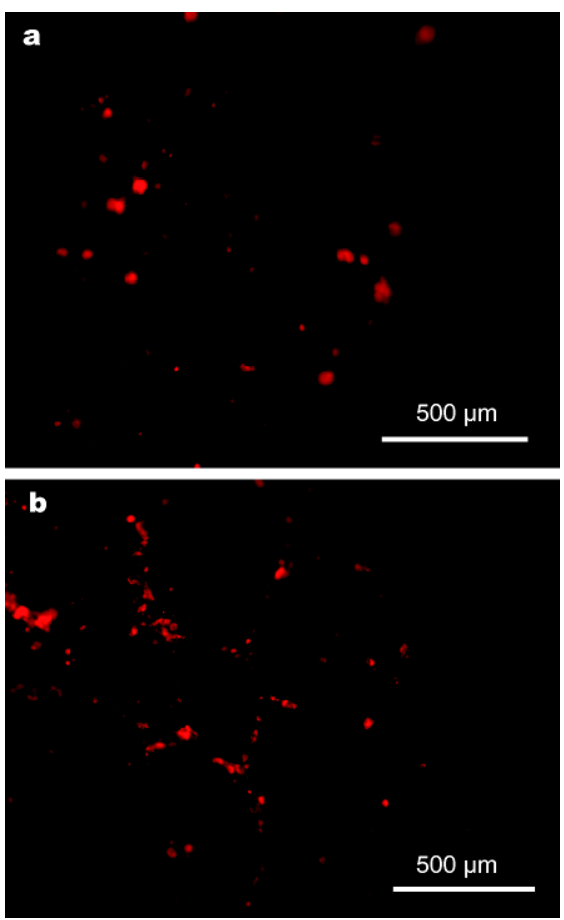

Figure 7 Human umbilical vein endothelial cells (HUVECs) forming vascular network with $2 \mathrm{mmol} \mathrm{L}^{-1}$ DFM treatment. (a) Negative control, no DFM; (b) DFM treatment.

Fig. 8, mature blood vessel development will require fibroblast cells in connective tissue to form the outer vessel structure and vascular smooth muscle cells to form the intermediate vessel structure. These cells can be included in the hydrogel matrix along with additional and appropriate growth factors (signaling molecules).

\section{Osseointegration and bone in-growth}

The animals survived the full study, with no evidence of infection. There were no signs of cage migration in either group at three or six months according to X-ray and micro $\mathrm{CT}$ imaging. At three months post-implantation, micro CT imaging and histological staining showed surrounding bone tissue growing into the porous $\mathrm{Ti}$-alloy cages and partially fusing the two vertebral bodies, although there still had a region of reduced radiolucency in the X-ray images and small empty gap in micro CT imaging (Fig. 9) around the cage edge. At six months post-surgery, the vertebral bodies had achieved complete spine fusion with the disappearance of the radiolucent region in X-ray images. Micro-CT showed a rapid ingrowth of bone tissue into the porous Ti-alloy cages from three to six months (an increase of BVF from $27.1 \% \pm 3.7 \%$ to $39.7 \% \pm 3.4 \%$ ). At six months the exterior perimeter of the porous Ti-alloy cage

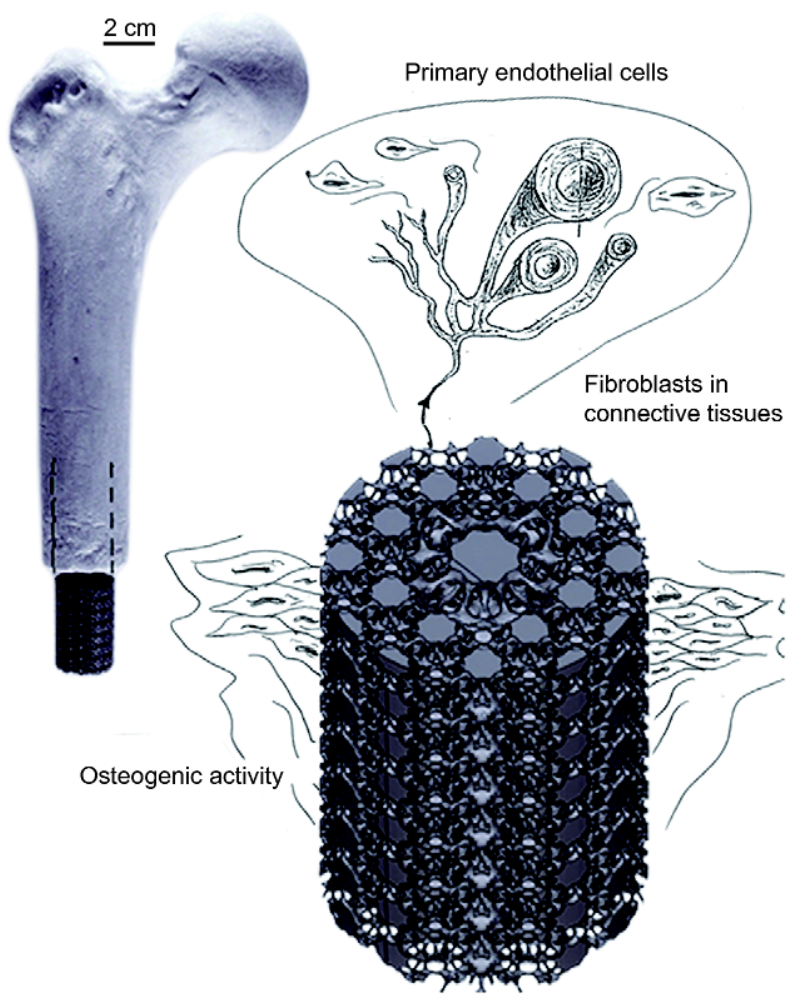

Figure 8 Schematic view of osseointegration and vascular network (blood vessel) function in porous EBM- fabricated intramedullary rod in the femur.

was covered with continuous bone, with increased bone tissue ingrowth and dramatically decreased cartilaginous and fibrous tissue. Unlike the indirect bone-material bonding at three months, the bone tissue bonded closely with the Ti-alloy cages, with most of the bone tissue found around the struts at interior and peripheral regions of the cages (Fig. 9). Consistent with the micro-CT findings, histomorphometric analysis showed a rapid ingrowth of bone tissue into the porous Ti cages (MBF increased from $22.2 \% \pm 3.2 \%$ at three months to $38.4 \% \pm 1.7 \%$ at six months).

The above results show good bone ingrowth and osseointegration in animal experiment. Based on our study and literature review, we suggest that surface properties and mechanical properties of the cage are both important factors for osseointegration. Recently, Ti alloy has been found to provide a highly favorable surface for osteoblast adhesion and osteogenic differentiation. Rapuano et al. [28] reported that Ti-alloy can form a superficial oxide layer which promoted osteoblast attachment through binding of adsorbed fibronectin to surface-expressed osteoblast integrins. Moreover, Olivares-Navarrete et al. [29] 


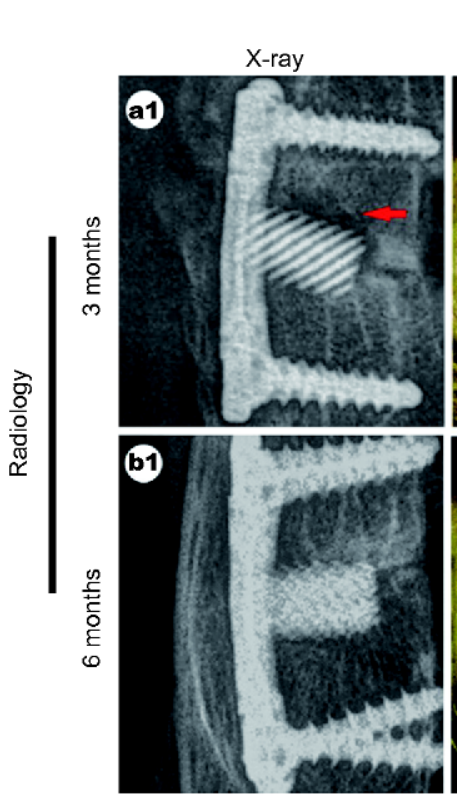

Overivew

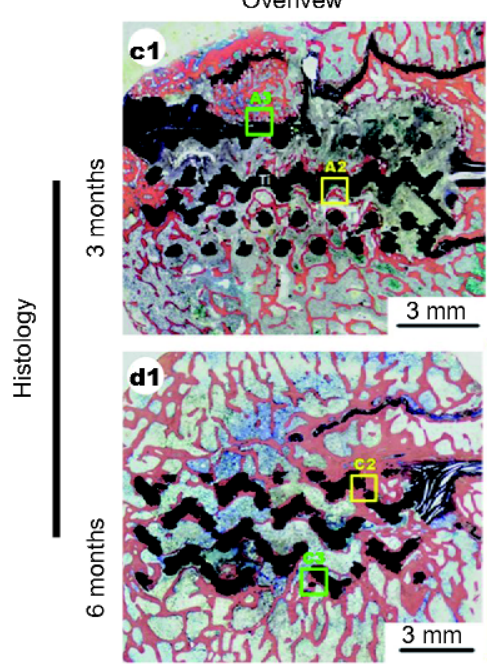

Micro-CT

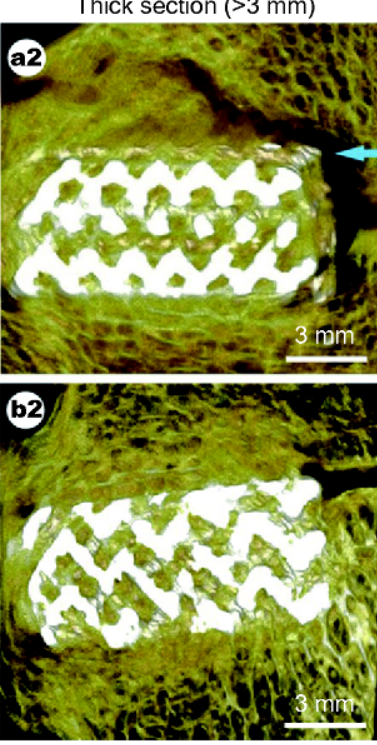

$\times 100$

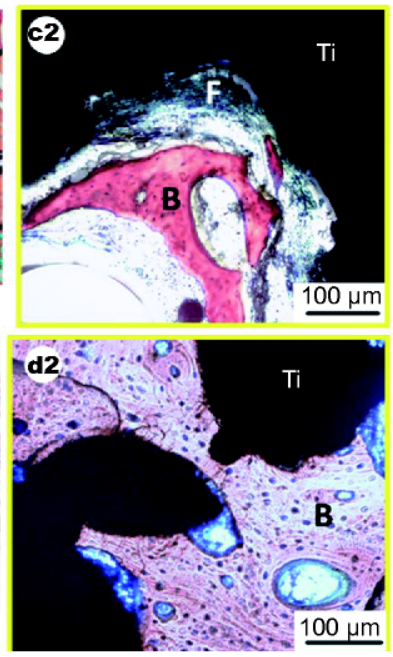

Micro-CT Thick section (<1 mm)

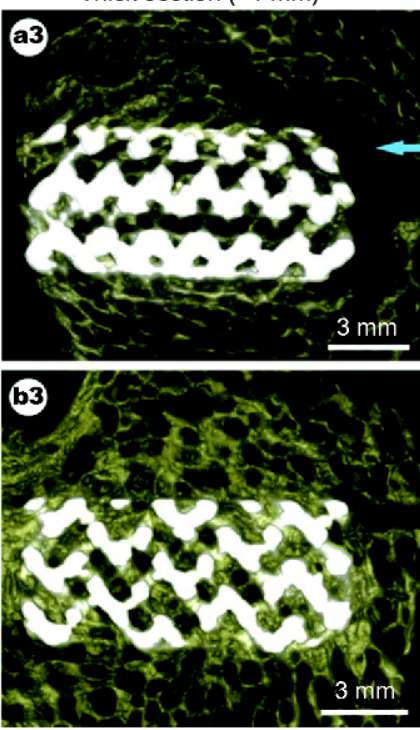

$\times 100$

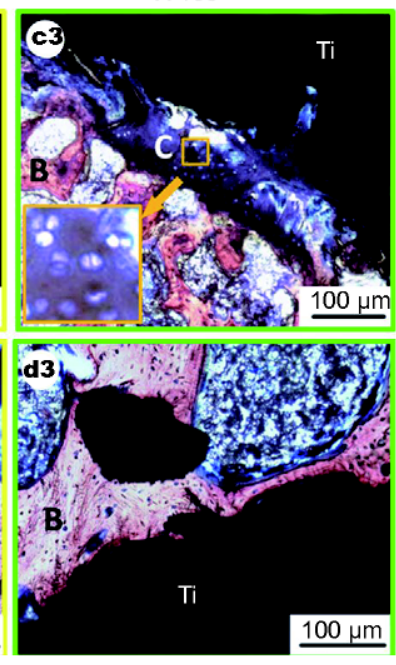

Figure 9 Lateral X-ray (a1, b1), micro-CT (a2-3, b2-3) and histological images (c1-3, d1-3) of porous Ti-alloy in the animal experiment. (a1-3) At 3 months, Ti-alloy cages had sectional osseointegration with surrounding bone tissue, as shown by the sectional radiolucent region and smaller empty gap (red arrow head) around the edge of the cages. (b1-3) At 6 months, Ti-alloy cages were well integrated with surrounding bone tissue and achieved a complete spine fusion, as shown by the close contact between bone and cage. (c1-3) At 3 months, bone matrix had infiltrated into the interconnected pores of porous Ti-alloy cages. Although most bone trabeculae did not directly bond to the Ti material surface (separated by a layer of fibrous tissue), intimate cartilage-material bonding was observed in some bone-material interface regions. (d1-3) At 6 months, most of the mature bone tissue bonded closely with the struts of porous Ti-alloy cages at both interior and peripheral regions of the cages. Bone (B), fibrous tissues (F), cartilage (C).

found that culture of MG 63 osteoblast-like cells on Ti$6 \mathrm{Al}-4 \mathrm{~V}$ can promote osteogenic maturation and create an osteogenic environment that could enhance bone formation and implant stability. Mechanical property of the implant is another factor which affects osseointegration. The elastic modulus of solid Ti-6Al-4V is $110 \mathrm{GPa}$, which is around 40- to 2000-times higher than that of natural bone tissue (0.05-30 GPa). This high modulus would lead to a stress shielding effect at the bone-implant interface, resulting in bone resorption and fibrous tissue encapsulation.

In the present study, the modulus of the Ti-alloy cage was decreased to $2.5 \mathrm{GPa}$ by adopting a porous structure, which matches the mechanical properties of natural bone tissue more closely. This modification led to improved metal-bone integration. Although the porous structure of 

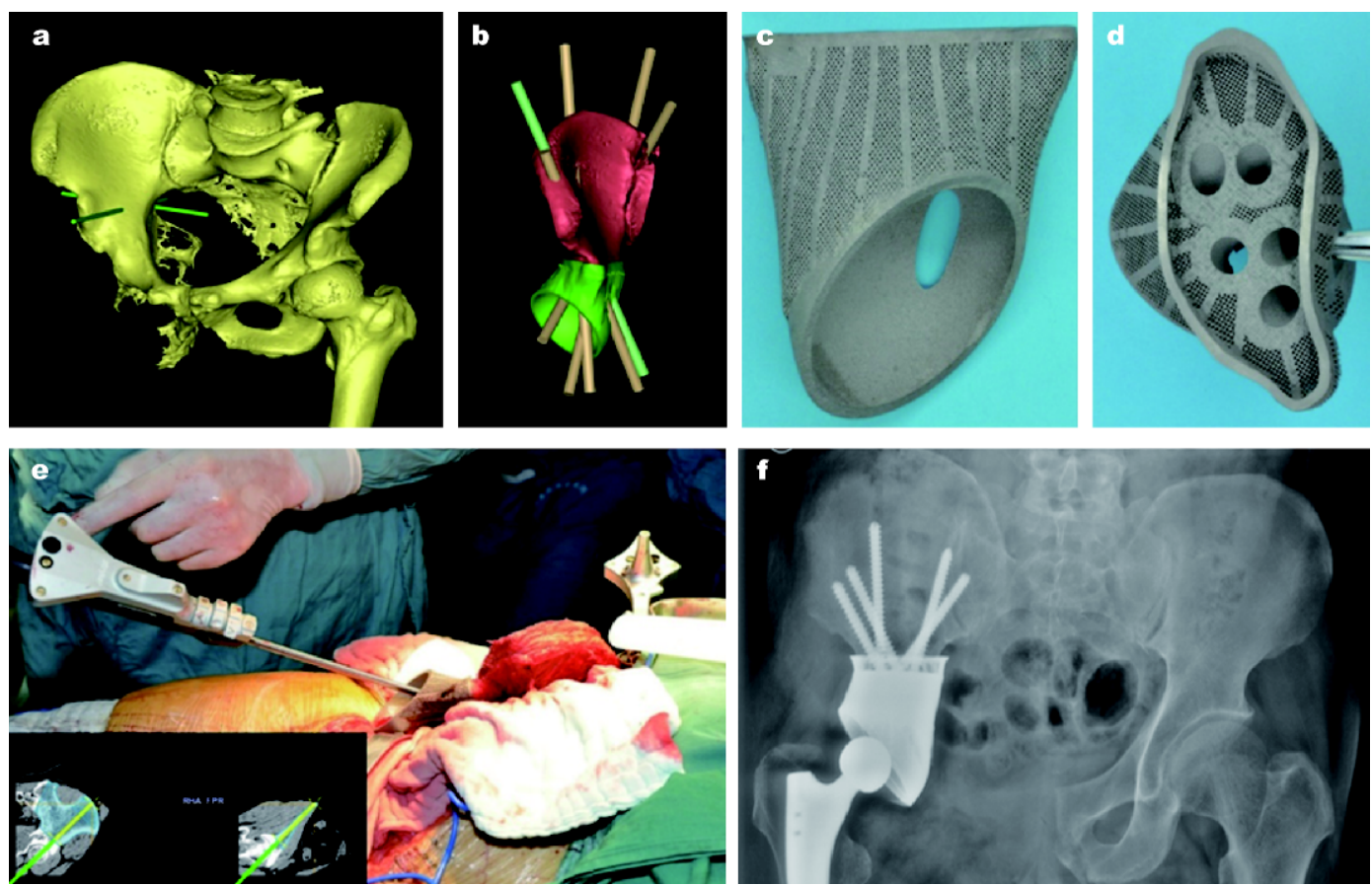

Figure 10 Surgical planning and results. (a) Reconstructed 3D pelvic tumor model. (b) The virtual model of acetabular prosthesis, simulated resection in a safe margin and prosthesis reconstruction with fastening screws. (c) Anterior of the custom-made Ti-alloy acetabular prosthesis by EBM. (d) The superior aspect of the prosthesis. (e) Implantation and fixation of the prosthesis aided by computer-aided navigation system. (f) X-ray film shows good alignment at 18 months postoperatively.

the Ti-alloy cage resulted in a low compressive strength (63 $\mathrm{MPa})$, this mechanical strength is sufficient for load bearing during intervertebral fusion, since the compressive strength of adjacent cancellous bone is only 2-12 MPa. This is further supported by the finding that neither compression nor fracture occurred in any porous Ti-alloy cage during the study. These two mechanisms are suggested to contribute to the good osseointegration of porous Ti-alloy cage.

\section{Human implant trials}

With GCTs that involve the acetabulum, it is essential to reconstruct the acetabulum after partial pelvic resection to obtain functional recovery. Although there are many options for the reconstruction, including flail hip, arthrodesis, allografting, and prosthetic replacement, many of them have poor functional results and high incidence of complications, due to the complex anatomy structure of the periacetabular.

In this case, a custom-made periacetabular prosthesis has been prepared and implanted precisely. Fig. 10 shows a good position and satisfactory fixation at 18 months postoperatively. According to MSTS 93 system for the upper and lower limb, the scores were 27 (90\%), which means a very good hip function. Customized prosthesis manufactured by rapid prototyping (RP) technique had been applied in limb salvage surgeries. Li et al. [30] designed a customized hemi-knee joint trying to solve the problem of cartilage necrosis in hemi-joint allograft transplantation. Dai et al. [31] reported their experience in customized hemi-pelvic prostheses implantation in ten patients who underwent internal hemi-pelvectomy for extensive pelvic tumors. However, the prostheses in these reports were still manufactured through mold-melted founding process. The RP technique was only used to fabricate positive mold. In the current study, the customized prosthesis was directly manufactured through EBM. Horn et al. [32] suggested that this direct metal fabrication technique can eliminate several process steps associated with traditional methods. Also, the traditional computer numerical control milling also has shortcomings when trying to replicate complex shape or internal features due to collision between the milling tool and the object. With the EBM technique, the accurate production of prosthesis with complicated morphology and internal features has become possible. In the present study, the customized prosthesis provides a precisely matching interface between host bone and implant. The internal porous structure has 
three primary advantages including decreasing weight of prosthesis, reducing the stress shielding effect and providing the space for bone ingrowth, which contribute to the good functional results and prosthetic survive.

The results of both in-vitro and in-vivo studies of $3 \mathrm{D}$ fabricated open-cellular (mesh and foam) implant structures demonstrate that not only can these implants be designed to reduce or eliminate stress shielding, but they can provide conditions that favor bone tissue formation, adhesion, proliferation and mineralization along with angiogenesis, all of which facilitate circulation of physiological fluids, nutrients, oxygen and cell distribution throughout the implant. Preliminary trials demonstrate the utility of these features which attest to the development of engineered (living) implants, and patient specific manufacture using EBM technologies.

\section{CONCLUSIONS}

Experimental observations of osseointegration in mesh and foam 3D Ti-6Al-4V structures indicate these structures are conducive to cellular functions involving osteoblast cell adhesion, proliferation, mineralization and protein synthesis. More porous (or lower density) structures (porosity $>75 \%$ ) corresponding to lower stiffness (and softer bone) exhibit less prolific cell growth or rate of proliferation in comparison to Mesh $2 \mathrm{~mm}$ (porosity $\sim 55 \%$ ), whereas cell seeding efficiency has shown a gradual decrease with increase in porosity, although all porosities investigated exhibited bioactivity leading to colonization of the entire porous structure. Adhering cells formed sheets bridging the pores and leading to intercellular interaction which provided a pathway for communication and maturation to a differentiated phenotype.

The seeding of Matrigel (a registered commercial hydrogel) with osteoblast and endothelial cell types infused into open-cellular Ti-6Al-4V structures, along with hypoxia-inducing DFM, produced primary vascular capillaries without degrading the osteoblast cell activity. Correspondingly, since both the Matrigel and the DFM are US Federal Drug Administration (FDA) approved materials, prospects for infusion of EBM-fabricated, open-cellular implant structures to promote osseointegration and vascularization appear promising.

Implant (human) trials of EBM-fabricated open-cellular Ti-6Al-4V structures indicate very favorable performance especially involving osseointegration. While vascular structure development has not been confirmed specifically by direct observation in human trials, the overall implant performance results would tend to confirm prospects for complete osseointegration and vascularization, leading to a "living implant" after a minimum of 6 months.

Received 28 April 2017; accepted 15 June 2017;

published online 4 August 2017

1 Maradit Kremers H, Larson DR, Crowson CS, et al. Prevalence of total hip and knee replacement in the united states. J Bone Joint Surgery-Am Volume, 2015, 97: 1386-1397

2 Murr LE, Gaytan SM, Medina F, et al. Next-generation biomedical implants using additive manufacturing of complex, cellular and functional mesh arrays. Philos Trans R Soc A-Math Phys Eng Sci, 2010, 368: 1999-2032

3 Koike M, Greer P, Owen K, et al. Evaluation of titanium alloys fabricated using rapid prototyping technologies-electron beam melting and laser beam melting. Materials, 2011, 4: 1776-1792

4 Sing SL, An J, Yeong WY, et al. Laser and electron-beam powderbed additive manufacturing of metallic implants: a review on processes, materials and designs. J Orthop Res, 2016, 34: 369-385

5 Bezuidenhout MB, Dimitrov DM, van Staden AD, et al. Titaniumbased hip stems with drug delivery functionality through additive manufacturing. BioMed Res Int, 2015, 2015: 1-11

6 Carmeliet P. Mechanisms of angiogenesis and arteriogenesis. Nat Med, 2000, 6: 389-395

7 Anderson CR, Ponce AM, Price RJ. Immunohistochemical identification of an extracellular matrix scaffold that microguides capillary sprouting in vivo. J Histochem Cytochem, 2004, 52: 10631072

8 Matena J, Petersen S, Gieseke M, et al. SLM produced porous titanium implant improvements for enhanced vascularization and osteoblast seeding. IJMS, 2015, 16: 7478-7492

9 Kumar A, Nune KC, Murr LE, et al. Biocompatibility and mechanical behaviour of three-dimensional scaffolds for biomedical devices: process-structure-property paradigm. Int Mater Rev, 2016, 61: $20-45$

10 Lieberman JR, Daluiski A, Einhorn TA. The role of growth factors in the repair of bone. J Bone Joint Surgery-Am Volume, 2002, 84: 1032-1044

11 Nune KC, Kumar A, Murr LE, et al. Interplay between self-assembled structure of bone morphogenetic protein-2 (BMP-2) and osteoblast functions in three-dimensional titanium alloy scaffolds: stimulation of osteogenic activity. J Biomed Mater Res, 2016, 104: 517-532

12 Kang HW, Lee SJ, Ko IK, et al. A 3D bioprinting system to produce human-scale tissue constructs with structural integrity. Nat Biotechnol, 2016, 34: 312-319

13 Petrovic V, Haro JV, Blasco JR, et al. Additive manufacturing solutions for improved medical implants. In: Lin C (Ed.), Biomedicine. Shanghai: In Tech China, 2012: 147-180

14 Li SJ, Murr LE, Cheng XY, et al. Compression fatigue behavior of Ti-6Al-4V mesh arrays fabricated by electron beam melting. Acta Mater, 2012, 60: 793-802

15 Gibson LJ, Ashby MF. Cellular Solids: Structural Properties. New York: Cambridge University Press, 1997

16 Wu SH, Li Y, Zhang YQ, et al. Porous titanium-6 aluminum-4 vanadium cage has better osseointegration and less micromotion than a poly-ether-ether-ketone cage in sheep vertebral fusion. Artif Organs, 2013, 37: E191-E201

17 Krock BL, Skuli N, Simon MC. Hypoxia-induced angiogenesis: good and evil. Genes Cancer, 2011, 2: 1117-1133 
18 Chen LL, Huang M, Tan JY, et al. PI3K/AKT pathway involvement in the osteogenic effects of osteoclast culture supernatants on preosteoblast cells. Tissue Eng Part A, 2013, 19: 2226-2232

19 Veschini L, Belloni D, Foglieni C, et al. Hypoxia-inducible transcription factor-1 alpha determines sensitivity of endothelial cells to the proteosome inhibitor bortezomib. Blood, 2007, 109: 25652570

20 Donneys A, Weiss DM, Deshpande SS, et al. Localized deferoxamine injection augments vascularity and improves bony union in pathologic fracture healing after radiotherapy. Bone, 2013, 52: 318325

21 Correa-Rodriguez V. Vascularization in interconnected 3D printed Ti-6Al-4V foams with hydrogel matrix for biomedical bone replacement implants. Doctoral Dissertation, El Paso, University of Texas at El Paso, 2016

22 Nune KC, Misra RDK, Gaytan SM, et al. Biological response of next-generation of 3D Ti-6Al-4V biomedical devices using additive manufacturing of cellular and functional mesh structures. J Biomater Tissue Eng, 2014, 4: 755-771

23 Nune KC, Misra RDK, Gaytan SM, et al. Interplay between cellular activity and three-dimensional scaffold-cell constructs with different foam structure processed by electron beam melting. J Biomed Mater Res, 2015, 103: 1677-1692

24 Karageorgiou V, Kaplan D. Porosity of 3D biomaterial scaffolds and osteogenesis. Biomaterials, 2005, 26: 5474-5491

25 Story BJ, Wagner WR, Gaisser DM, et al. In vivo performance of a modified CS Ti dental implant coating. Int J Oral Maxillofac Implants, 1998, 13: 749-757

26 le Noble F, le Noble J. Bone biology: vessels of rejuvenation. Nature, 2014, 507: 313-314

27 Ramasamy SK, Kusumbe AP, Wang L, et al. Endothelial notch activity promotes angiogenesis and osteogenesis in bone. Nature, 2014, 507: 376-380

28 Rapuano BE, Lee JJE, MacDonald DE. Titanium alloy surface oxide modulates the conformation of adsorbed fibronectin to enhance its binding to $\alpha 5 \beta 1$ integrins in osteoblasts. Eur J Oral Sci, 2012, 120: 185-194

29 Olivares-Navarrete R, Gittens RA, Schneider JM, et al. Osteoblasts exhibit a more differentiated phenotype and increased bone morphogenetic protein production on titanium alloy substrates than on poly-ether-ether-ketone. Spine J, 2012, 12: 265-272

$30 \mathrm{Li} \mathrm{Z}, \mathrm{Xu} \mathrm{SF}, \mathrm{Li} \mathrm{DC}$, et al. Composite artificial semi-knee joint system. Eur Rev Med Pharmacol Sci, 2014, 18: 1229-1240

31 Dai KR, Yan MN, Zhu ZA, et al. Computer-aided custom-made hemipelvic prosthesis used in extensive pelvic lesions. J Arthroplasty, 2007, 22: 981-986

32 Horn TJ, Harrysson OLA. Overview of current additive manufacturing technologies and selected applications. Sci Prog, 2012, 95: $255-282$

Acknowledgements This research was supported in part by the Ministry of Science and Technology Project (2017YFC1104900 and 2016YFC1102601) and the National Natural Science Foundation of China (51271182 and 51631007), Chinese Academy of Sciences Project (QYZDJ-SSW-JSC031), the Department of Metallurgical, Materials and Biomedical Engineering (Nune KC and Misra RDK), and the Office of Research and Sponsored Projects (Correa-Rodriguez VL and Murr LE) at the University of Texas at El Paso.

Author contributions Li S, Guo Z, Misra RDK and Murr LE designed the experiments. Nune $\mathrm{KC}$ and Correa-Rodriguez VL carried out in vitro experiments. Guo $\mathrm{Z}$ and $\mathrm{Li} \mathrm{X}$ carried out the animal and clinical experiments. Li $\mathrm{S}$ and Hou $\mathrm{W}$ fabricated all specimens. The paper was written by Li S, Li X, Misra RDK and Murr LE. The project was supervised by Hao Y and Yang R. All authors contributed to the discussion and data analyses.

Conflict of interest The authors declare that they have no conflict of interest. 

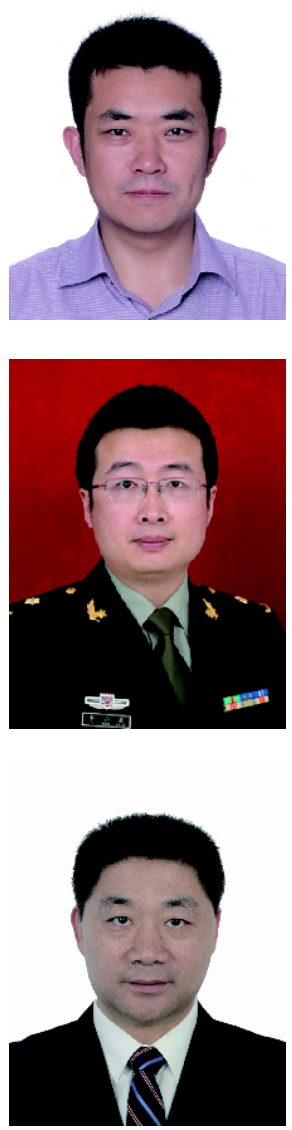

Shujun Li is a tenured professor in Shenyang National Laboratory for Materials Science, Institute of Metal Research, Chinese Academy of Sciences. His current research interest focuses on additive manufacturing via electron beam melting technology, aiming to understand the structure-process-property relationship of 3D printable metallic materials and explore their practical applications in aerospace and biomedical components, etc.

Xiaokang Li, MD, PhD, is an attending physician at the Department of Orthopaedic Oncology, Xijing Hospital. His research interest focuses on the effect of bone implants made of low modulus titanium or porous titanium alloy.

Zheng Guo is a professor and Chief of the Department of Orthopaedic Oncology, Xijing Hospital. His clinical work has focused on the application of customized 3D-printed titanium implants and digital orthopedic techniques. His research interests have been in both clinical and basic areas. The clinical research involves modifying surgical techniques and R\&D of new orthopedic implants. His basic research focuses on low modulus titanium alloy, porous titanium alloy and surface modification for metal materials.

\section{钛合金多孔植入物: 骨整合、血管化和临床实验}

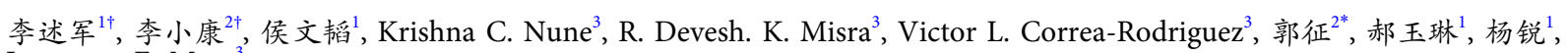
Lawrence E. Murr

摘要 本文采用电子束增材 $(\mathrm{EBM})$ 制造技术制备了多种具有开放孔隙结构的多孔钛合金 (Ti-6Al-4V)植入物, 包括网状和泡沫状结构. 该多孔钛 合金植入物可以通过调节孔隙率(或密度)降低其弹性模量(或刚度)以减轻 “应力屏蔽” 效应，实现与软(小梁)和硬(皮质)骨的弹性模量(或刚 度)匹配; 同时还可以促进骨组织长入，增加细胞密度和细胞外基质间的相互作用，后者涉及骨形态发生蛋白(BMP-2)和成骨细胞功能之间的 相互影响. 总结了在水性水凝胶基质中初级血管结构的早期形成和特征，报道了EBM技术制备的个性化钛合金植体在动物(羊)和人体临床试 验的初步结果. 本文结果为钛合金多孔材料作为组织工程 “活性” 植入物的应用可行性研究提供了有力支持. 